\title{
INFLUÊNCIA DE INDICADORES SOCIOECONÔMICOS NO CONSUMO PER CAPITA DE ÁGUA PARA OS MUNICÍPIOS DO ESTADO DA BAHIA
}

\author{
Influence of socioeconomic indicators on per capita water \\ consumption for municipalities in the state of Bahia, Brazil \\ Juliane Ferreira Caldeira, Davi Santiago Aquino \\ Instituto Federal de Educação, Ciência e Tecnologia da Bahia - IFBA, campus Eunápolis, \\ BA. \\ E-mail:_julianecaldeira@ifba.edu.br; davi.aquino@ifba.edu.br
}

RESUMO - Fatores socioeconômicos podem influenciar os aspectos quantitativos dos serviços inerentes aos componentes do saneamento básico. Esta pesquisa consiste na aplicação da correlação linear de Pearson entre consumo de água potável e parâmetros socioeconômicos, ao mensurar e classificar a influência do índice de desenvolvimento humano municipal e do produto interno bruto per capita no consumo médio per capita de água. Analisaram-se as correlações utilizando dados dos 417 municípios do estado da Bahia, Brasil, abrangendo o intervalo temporal entre os anos de 2002 e 2016. Os resultados obtidos demonstram a ocorrência de uma correlação linear de Pearson classificada como fraca positiva entre o índice de desenvolvimento humano e o consumo de água e de uma correlação classificada como fraca negativa entre o produto interno bruto e o consumo per capita de água, para a maioria dos municípios analisados. As características operacionais das ferramentas utilizadas mostraram-se eficientes, simples e de relevante utilidade para elaboração de estudos destinados ao planejamento e à melhoria dos sistemas de abastecimento de água.

Palavras-chave: Abastecimento de Água; Correlação de Pearson; Dados Municipais.

ABSTRACT - Socioeconomic factors can influence the quantitative aspects of services that are inherent to basic sanitation components. This study consists of the application of the Pearson correlation between drinking water consumption and socioeconomic parameters in order to measure and classify the influence of the municipal human development index and the per capita gross domestic product on per capita water consumption. Correlations were analyzed using data from 417 municipalities in the state of Bahia, Brazil from 2002 to 2016. The results demonstrate the occurrence of a weakly positive Pearson linear correlation between the human development index and water consumption and a weakly negative correlation between the gross domestic product and the per capita water consumption for most of the analyzed municipalities. The operational characteristics of the tools used here proved to be efficient, simple, and important for building studies aimed toward the planning and improvement of water supply systems.

Keywords: Water Supply; Pearson Coefficient; Municipal Data. 


\section{INTRODUÇÃO}

A eminência do saneamento e sua associação com a saúde pública acompanham o desenvolvimento das civilizações humanas desde sua origem (FUNASA, 2019). No Brasil, a Política Nacional de Saneamento Básico, instituída pela Lei Federal no. 11.445 de 05 de janeiro de 2007, descreve o saneamento como o conjunto de serviços, infraestruturas e instalações operacionais relativos a abastecimento de água, esgotamento sanitário, limpeza urbana e manejo de resíduos sólidos e a drenagem e manejo de águas pluviais (BRASIL, 2007).

Embora esses quatro componentes sejam intrinsecamente correlatos entre si, e, portanto, necessários em sua conjuntura para o bem estar da sociedade, um enfoque específico pode ser direcionado aos serviços públicos de abastecimento de água potável.

O adequado suprimento de água possui papel primordial para a sobrevivência e o desenvolvimento humanos. Porém, a disponibilidade desse recurso natural tem sido insuficiente para atender à demanda requerida em diversas regiões do planeta. Assim, para minimizar o agravamento dos efeitos desse fenômeno, as instalações para abastecimento de água devem ser capazes de fornecer água potável com qualidade, visando priorizar a proteção da saúde humana, com regularidade e de forma acessível para as populações. Ademais, devese atender ainda à meta da universalização e ao princípio da equidade de acesso aos mananciais por parte de todos os usuários, da presente e de futuras gerações (HELLER, 2010).

Dados consolidados do território nacional para o ano base de 2018 mostram o atendimento de abastecimento de água para 169,1 milhões de brasileiros, correspondendo a $83,6 \%$ da população. Esse panorama nacional apresenta ainda a existência de 662,6 mil quilômetros de redes de distribuição de água potável, 57,2 milhões de ligações de água e um investimento anual de $\mathrm{R}$ 5,75 bilhões (SNIS, 2020a).
A titularidade dos serviços de abastecimento público de água é cabível às municipalidades, que podem delegar a operação desse sistema, conforme preconizado no Artigo 241 da Constituição Federal (BRASIL, 1988).

No panorama nacional, dos 1.151 prestadores de serviço responsáveis pelo abastecimento público de água, 51,0\% pertencem à administração pública direta, $37,0 \%$ são autarquias, $8,6 \%$ são empresas privadas e o percentual restante corresponde a atuação de sociedades de economias mistas, empresas públicas e organizações sociais (SNIS, 2020a). Esses prestadores de serviços têm a obrigação de suprir a população com uma água sanitariamente segura, que no Brasil para ser considerada como potável precisa atender aos padrões de potabilidade estabelecidos pelo Anexo XX da Portaria de Consolidação n. 005 de 2017 do Ministério da Saúde (BRASIL, 2017).

Nesse sentido, o planejamento e a gestão dos sistemas públicos de abastecimento de água podem reduzir diversos impactos negativos na infraestrutura construída, no meio ambiente e na saúde, além de serem indicativos de qualidade de vida e de fomentar a economia de um município. Porém, além de variáveis técnicas para o dimensionamento e a operação desses sistemas, é indispensável uma análise mais abrangente, incluindo parâmetros indiretos do potencial hídrico, como variáveis socioeconômicas (HELLER, 2010).

Diversos índices socioeconômicos têm sido utilizados na obtenção de modelagens matemáticas que sejam capazes de fazer predição de fatores quantitativos correlatos a planejamento e expansão dos serviços públicos de abastecimento de água (SILVA et al., 2008; ADAMS; PABLOS, 2010).

Dois desses indicadores socioeconômicos municipais que podem ter relação direta com indicadores relativos aos serviços de abastecimento de água são o Índice de Desenvolvimento Humano Municipal (IDHM) e o Produto Interno Bruto (PIB), visto que se pode levantar a hipótese 
da existência da correlação entre os indicadores de saneamento com parâmetros socioeconômicos, em especial entre o consumo per capita de água e indicadores de desenvolvimento social e econômico (LIBÂNIO;CHERNICHARO; NASCIMENTO, 2005).

No âmbito internacional, FernándezDurán e Lloret (2016) analisaram a relação entre PIB per capita e consumo de água para os 131 municípios pertencentes à bacia hidrográfica Lerma-Chapada, localizada na região central oeste do México e obtiveram como resultado um modelo estatisticamente significativo, pelo método da curva em $U$ invertida de Kuznets, relacionando as referidas variáveis.

Já no Brasil, Fernandes Neto et al. (2005) analisaram dados relativos a 96 municípios de Minas Gerais com população abaixo de 300 mil habitantes e obtiveram coeficientes de determinação estatisticamente significativos para correlações feitas para se averiguar a influência da renda per capita e do IDHM no consumo per capita de água.

Ademais, estudo realizado nos 246 municípios do estado de Goiás analisou a relação de dados correlatos a saneamento básico, índice de desenvolvimento humano e cobertura vegetal e atestou que municípios com maior quantidade de domicílios com saneamento adequado possuíam proporcionalmente maiores valores de IDHM (SOUZA; FERREIRA; FORMIGA, 2016). Ainda em Goiás, mas em 26 municípios da região sudoeste do estado, dados municipais de PIB per capita e de IDHM foram analisados via correlação de Pearson quanto às suas respectivas influências no consumo de água por habitante, observando-se a ocorrência de correlação positiva entre PIB e consumo de água para a maioria dos municípios, além de fraca influência do IDH no referido consumo per capita (AQUINO et al., 2017).

O coeficiente da correlação linear de Pearson é uma metodologia estatística capaz de mensurar de forma adimensional o sentido e o grau de relacionamento linear entre duas variáveis aleatórias quantitativas, visto que seus valores situam-se entre os extremos de -1,0 e +1,0 (WASSERMAN, 2004; FIGUEIREDO FILHO; DA SILVA JÚNIOR, 2009; BONAMENTE, 2017). A obtenção do referido coeficiente pode ocorrer manualmente ou pela utilização de programas computacionais específicos de estatística ou de planilhas eletrônicas, como o Microsoft Excel ${ }^{\circ}$ (GUERRERO, 2019).

Até o presente momento, não foram identificados estudos que avaliaram a influência de índices socioeconômicos no consumo de água para os municípios do estado da Bahia, seja pela correlação de Pearson ou por outro recurso estatístico. Logo, a existência de resultados advindos de uma análise estatística de possível correlação entre consumo de água e índices socioeconômicos pode fornecer subsídios a futuros estudos de planejamento, tais como ampliação da capacidade de operação de estações de tratamento de água e de esgotos ou da extensão de redes de abastecimento de água e de coleta de esgotos, visando a melhoria do bem estar social e econômico da população dos municípios baianos.

Nesse contexto, o presente trabalho objetivou aplicar a análise da correlação linear de Pearson para mensurar a influência dos indicadores socioeconômicos municipais IDH e PIB no consumo per capita de água para os 417 municípios do estado da Bahia, utilizando dados referentes aos anos de 2002 a 2016.

\section{MATERIAL E MÉTODOS}

\section{1. Área de estudo}

A presente pesquisa teve como área de estudo o estado da Bahia, que foi categorizado em seus municípios e em suas mesorregiões, segundo os procedimentos metodológicos explanados na sequência.

De acordo com os dados do Censo Demográfico de 2010, a Bahia é o quarto estado mais populoso do Brasil, sendo superado nesse quesito apenas por São Paulo, Minas Gerais e pelo Rio de Janeiro. Dos 14.016.906 residentes no estado 
contabilizados pelo referido Censo, 10.102.476, ou 72,1\%, residiam em área urbana. Ademais, para o ano de 2019, a população do estado foi estimada em 14.873.064 habitantes (IBGE, 2020a).

O estado é composto por 417 municípios, divididos em sete mesorregiões, quais sejam: Centro norte da Bahia, Centro sul baiano, Extremo oeste baiano, Metropolitana de Salvador, Nordeste baiano, Sul baiano e Vale São-Francisco da Bahia (IBGE, 2020a). A divisão do estado em suas mesorregiões é apresentada espacialmente na Figura 1, na qual utilizou-se WGS 84 como sistema de referência de coordenadas.

Figura 1. Mesorregiões do estado da Bahia.

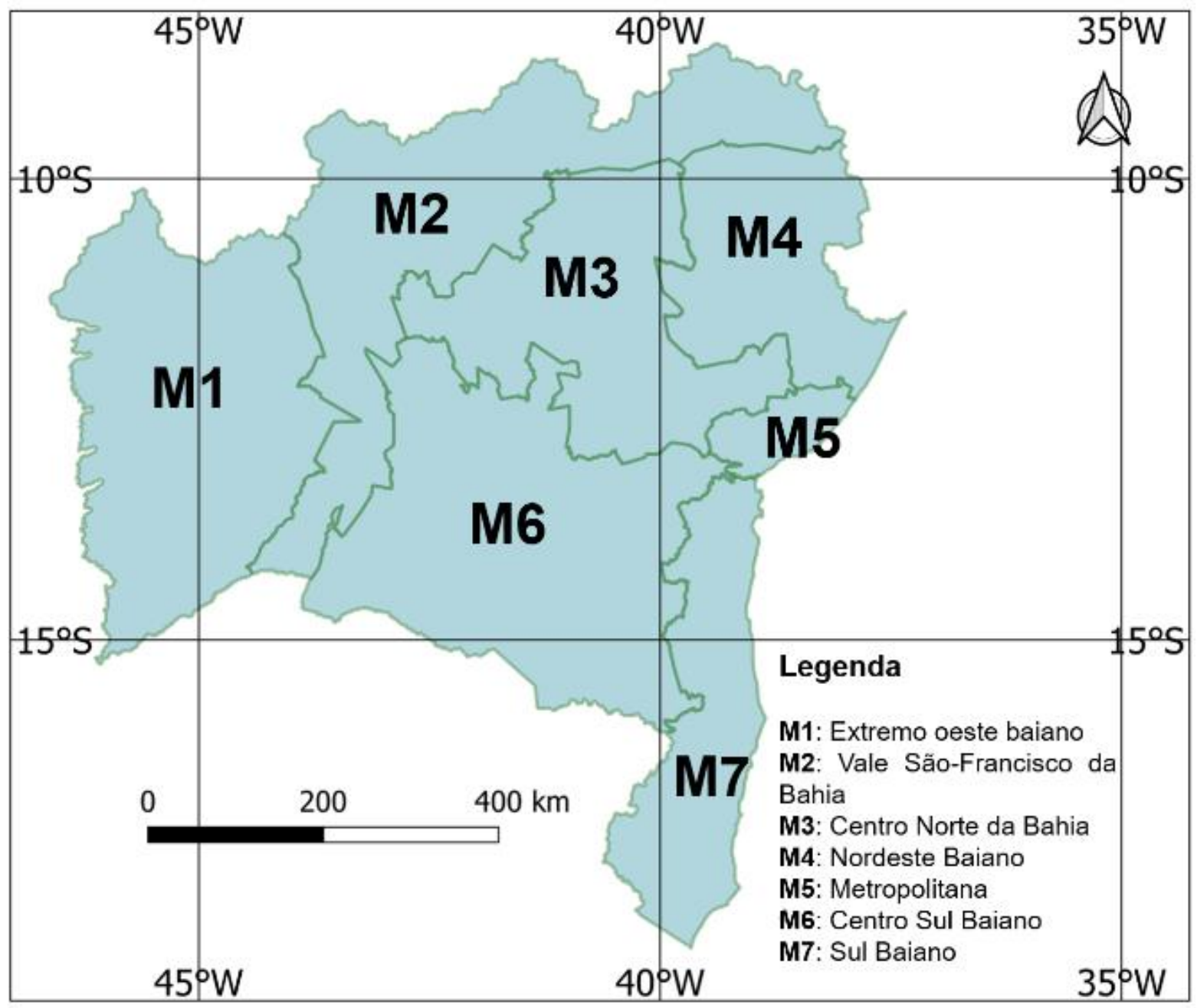

Fonte: O autor, com base cartográfica extraída de IGEO (2020).

De acordo com levantamento realizado pela Agência Nacional de Águas (ANA), as sedes urbanas do estado são abastecidas em sua maioria por mananciais superficiais, correspondendo

a
aproximadamente $73 \%$ dos municípios baianos, as águas subterrâneas somam $19 \%$ e os $8 \%$ dos municípios restantes são abastecidos por sistemas mistos. Todavia, o mesmo órgão alerta, embasando-se em dados relativos ao ano de 2015 , que apenas
22\% dos municípios do estado da Bahia são atendidos com um abastecimento satisfatório, enquanto $66 \%$ requerem ampliação do sistema de abastecimento de água e $12 \%$ necessitam de um novo manancial para suprir a demanda de água (ANA, 2020).

$\mathrm{Na}$ Bahia, cerca de $84 \%$ dos municípios, possuem seu abastecimento de água operado pela Empresa Baiana de Águas e Saneamento S.A. (Embasa), que é uma 
sociedade de economia mista de capital autorizado, cujo acionista majoritário é o Governo do Estado da Bahia (ANA, 2020).

\subsection{Dados utilizados}

Como informações relativas ao perfil socioeconômico dos municípios, utilizaram-se o Índice de Desenvolvimento Humano Municipal (IDHM) e o Produto Interno Bruto (PIB) de cada município do estado. Já em relação ao consumo de água, utilizaram-se as médias municipais anuais do consumo por habitante, disponibilizadas pelo Sistema Nacional de Informações sobre Saneamento (SNIS). O período abrangido pela presente pesquisa foi do ano de 2002 a 2016.

O Programa das Nações Unidas para o Desenvolvimento (PNUD) descreve o Índice de Desenvolvimento Humano Municipal como uma medida adimensional, variando de 0 a 1 e quanto mais próximo de 1 for o seu valor, maior será o desenvolvimento humano do município. O IDHM é composto pelas suas três dimensões básicas: educação, renda e longevidade e fornece um contraponto ao Produto Interno Bruto, que abrange apenas a dimensão econômica do desenvolvimento. 0 Índice de Desenvolvimento Humano Municipal difere do Índice de Desenvolvimento Humano Global, pois enquanto esse é calculado anualmente pelo escritório global da PNUD, o IDHM sofre um ajuste metodológico, adaptando-se à realidade do país, e é calculado para cada município (PNUD, 2020).

No Brasil, a disponibilização dos valores de IDHM acompanha as realizações do Censo Demográfico. Portanto, seu valor mais recente é referente ao ano de 2010. O cálculo desse indicador socioeconômico é realizado em conjunto pelas instituições PNUD Brasil, Instituto de Pesquisa Econômica Aplicada e a Fundação João Pinheiro e sua metodologia de cálculo baseia-se na média geométrica dos valores relativos aos seus três componentes: IDHM Longevidade, IDHM Educação e IDHM Renda (ATLAS BRASIL, 2020).
Já o PIB per capita é um indicador síntese da economia, calculado comumente no período de um ano, com base no somatório de bens e serviços finais de um país, estado ou cidade e dividido pelo número de habitantes, estipulando quanto cada indivíduo receberia caso o PIB fosse dividido em partes iguais. Desta forma, embora expresse a riqueza de um território ao ser um indicador síntese de uma economia, o PIB per capita não exprime outros importantes fatores inerentes à complexidade socioeconômica, como distribuição de renda, qualidade de vida, educação e saúde (IBGE, 2020b).

O consumo médio per capita de água (QPC) é o volume médio diário de água consumida dividido pela população abastecida e é geralmente expresso $\mathrm{L}^{-1}$ hab $^{-1}$ $\mathrm{dia}^{-1}$ (VON SPERLING, 2014). No ano de 2018, o QPC médio do Brasil foi de $154,9 \mathrm{~L}^{-1} \mathrm{hab}^{-1}$ dia $^{-1}$, embora haja naturalmente variações regionais, visto que a média da região Nordeste no ano foi de 115,4 e a da região Sul foi de 182,6 $\mathrm{L}^{-1} \mathrm{hab}^{-1} \mathrm{dia}^{-1}$. Especificamente para o estado da Bahia, este indicador no mesmo ano de 2018 apresentou valor médio de $115,6 \mathrm{~L}^{-1} \mathrm{hab}^{-1} \mathrm{dia}^{-1}$ (SNIS, 2020a).

Os valores do IDHM referentes ao Censo Demográfico de 2010 de cada um dos 417 municípios baianos foram obtidos por intermédio da plataforma virtual do Atlas do Desenvolvimento Humano no Brasil (ATLAS BRASIL, 2020). Os valores municipais de PIB per capita o foram pela utilização da base de dados da Superintendência de Estudos Econômicos e Sociais da Bahia (SEI, 2020). Por sua vez, os valores do consumo médio per capita de água, cujo código de identificação é IN022_AE, foram obtidos no aplicativo Série Histórica, que contém uma base de dados disponível a consulta e é integrante do Sistema Nacional de Informações sobre Saneamento, vinculado à Secretaria Nacional de Saneamento do Ministério do Desenvolvimento Regional (SNIS, 2020b). Enquanto os valores de IDHM foram obtidos apenas para o ano de 2010, 
obtiveram-se os valores das outras variáveis para a série histórica contemplada entre os anos de 2002 a 2016.

Os dados obtidos foram sistematizados por município e por ano de referência, além de categorizados pelas suas variáveis: consumo médio per capita de água $\left(\mathrm{L}^{-1} \mathrm{hab}^{-1} \mathrm{dia}^{-1}\right)$, PIB per capita (R\$) e IDHM, com o uso de planilhas eletrônicas do programa computacional Microsoft Excel ${ }^{\circledR}$. Esse mesmo software foi utilizado para análise de estatística descritiva dos resultados.

\subsection{Análise estatística}

Realizaram-se dois tipos de análise de correlação linear de Pearson, a fim de se quantificar a influência de índices socioeconômicos no consumo de água: a primeira foi realizada entre IDHM 2010 e consumo per capita para o mesmo ano em todos os municípios do estado, categorizados por mesorregião. Dessa forma, essa primeira correlação não abrangeu uma série histórica, visto que não se possuem valores anuais de IDHM.

A segunda análise foi mais abrangente, pois para cada município realizou-se uma correlação linear de Pearson entre os valores anuais de PIB per capita e os respectivos consumos per capita de água entre os anos de 2002 e 2016. Por contemplar os 417 municípios do estado, esta segunda abordagem também foi analisada em mesorregiões do estado, visto que uma discussão específica para cada município seria impraticável.

Em ambas as abordagens foi utilizada a correlação linear de Pearson. Conforme já explanado, essa correlação expressa tanto a direção quanto o grau de relação linear existente entre duas variáveis quantitativas (BONAMENTE, 2017). Esse grau de relação varia de -1 a +1 e sua metodologia de cálculo algébrico é dada pela Equação 1.

$$
r=\frac{\sum\left(x_{i}-\bar{x}\right)\left(y_{i}-\bar{y}\right)}{\sqrt{\sum\left(x_{i}-\bar{x}\right)^{2}\left(y_{i}-\bar{y}\right)^{2}}}
$$

Na qual:

r: Coeficiente de Pearson;

$\mathrm{x}$ : Variável quantitativa independente;

$\overline{\mathrm{x}}$ : Média dos valores analisados da variável $\mathrm{x}$;

y: Variável quantitativa dependente;

$\bar{y}$ : Média dos valores analisados da variável y.

Os valores de coeficiente de Pearson foram obtidos pela aplicação da Equação 1 no programa computacional Microsoft Excel ${ }^{\circledR}$ (GUERRERO, 2019).

Na primeira forma de abordagem da relação entre consumo de água e variáveis socioeconômicas nos municípios da Bahia, a variável independente, $x$, foi o IDHM 2010 de cada um dos 417 municípios, enquanto a dependente, $y$, foi o respectivo consumo per capita médio diário de água dos mesmos municípios para o referido ano. Como essa abordagem metodológica foi categorizada por cada uma das sete mesorregiões do estado, obtiveram-se sete valores do coeficiente $r$ de Pearson, cada um quantificando o grau e a direção de correlação entre IDHM e consumo de água no ano de 2010 para os respectivos municípios de cada mesorregião.

Com alusão à abordagem na qual objetivou-se avaliar a correlação linear entre a variável independente PIB per capita e a dependente consumo per capita de água, realizou-se uma análise de correlação para cada município, visto que essas duas variáveis quantitativas apresentam valores anuais e a presente pesquisa abrangeu o período compreendido entre 2002 e 2016.

Por se tratar de uma análise de correlação, a obtenção do coeficiente de Pearson é influenciada pela quantidade de pares de dados e quanto menor a quantidade de pares disponíveis, menor é a confiabilidade do coeficiente obtido (FIGUEIREDO FILHO; DA SILVA JÚNIOR, 2009). Assim, os municípios que apresentaram menos de cinco pares de dados PIB versus consumo de água foram descartados da análise (WASSERMAN, 2004).

Como os valores do coeficiente de Pearson ( $r$ ) variam de -1 a +1 , é possível se 
aferir não apenas acerca do grau de correlação linear entre duas variáveis quantitativas, mas também quanto ao seu sentido. Assim, caso se obtenha, por exemplo, um valor de $r=1$ entre duas variáveis, significa que ao dobrar o valor da variável independente, observar-se-á, por conseguinte, um incremento de $100 \%$ na variável dependente.

Dessa forma, os valores desse coeficiente são alvo de distintas classificações, de acordo com a área de estudo. $\mathrm{Na}$ presente pesquisa, a interpretação dos valores do coeficiente de correlação linear de Pearson foi realizada com embasamento na classificação elaborada por Santos (2007) e apresentada na Tabela 1.

Tabela 1. Classificação da correlação linear do coeficiente de Pearson.

\begin{tabular}{c|l}
\hline Coeficiente de correlação & \multicolumn{1}{c}{ Correlação } \\
\hline$r=1$ & Perfeita positiva \\
\hline $0,8 \leq r<1$ & Forte positiva \\
\hline $0,5 \leq r<0,8$ & Moderada positiva \\
\hline $0,1 \leq r<0,5$ & Fraca positiva \\
\hline $0 \leq r<0,1$ & Ínfima positiva \\
\hline 0 & Nula \\
\hline$-0,1<r<0$ & Ínfima negativa \\
\hline$-0,5<r \leq-0,1$ & Fraca negativa \\
\hline$-0,8<r \leq-0,5$ & Moderada negativa \\
\hline$-1<r \leq-0,8$ & Forte negativa \\
\hline$r=-1$ & Perfeita negativa \\
\hline
\end{tabular}

Fonte: adaptado de Santos (2007).

A categorização desenvolvida por Santos (2007) para os valores do coeficiente da correlação linear de Pearson tem sido utilizada em pesquisas relacionadas a análises qualitativas e quantitativas de águas no Brasil, como nos estudos sobre qualidade das águas subterrâneas em poços no Estado do Ceará (COSTA et al., 2012) e sobre a avaliação físico-química e microbiológica das águas superficiais da bacia hidrográfica do rio Paraíso Jataí, no estado de Goiás (ROCHA et al., 2019).

Para auxiliar na interpretação dos resultados e subsidiar inferências inerentes, os valores do coeficiente $r$ da correlação linear de Pearson entre PIB e consumo de água entre 2002 e 2016 de cada município do estado, bem como sua classificação de acordo com a Tabela 1 e sua divisão pelas respectivas mesorregiões, foram representados espacialmente em mapa temático desenvolvido do programa computacional QGIS versão 3.10.3, que é um software multiplataforma de Sistema de Informações Geográficas de uso gratuito e com código fonte aberto.

\section{RESULTADOS E DISCUSSÃO}

\subsection{Influência do IDHM 2010 no consumo per capita de água}

Quanto à análise de correlação linear de Pearson entre o Índice de Desenvolvimento Humano Municipal e o consumo per capita de água no ano de 2010, categorizada pelas mesorregiões do estado, dos 417 municípios baianos, 24 não apresentaram valor de consumo per capita de água na base de dados do Sistema Nacional de Informações sobre Saneamento e, portanto, foram excluídos dessa análise.

Desses 24 municípios que não integraram as análises de correlação de Pearson entre IDHM 2010 e consumo per capita, 3 localizam-se na mesorregião Extremo oeste baiano: Cocos, Correntina e Jaborandi; 10 no Vale São-Francisco da Bahia: Buritirama, Campo Alegre de Lourdes, Casa Nova, Itaguaçu da Bahia, Macururé, Pilão Arcado, Rodelas, Sento Sé, Serra do Ramalho e Sítio do Mato; 1 no Centro Norte da Bahia: Pindobaçu; 7 no Centro Sul Baiano: Abaíra, Belo Campo, Boquira, Matina, Piatã, Riacho de Santana e Sebastião Laranjeiras e mais 3 no Sul baiano: Barro Preto, Gandu e Jussari.

Assim, a quantidade de pares de dados utilizados para se aferir o grau de influência no IDHM no consumo per capita de água em cada mesorregião do estado foi variável, de acordo como se apresenta na Tabela 2. 
Tabela 2. Correlação de Pearson entre o Índice de Desenvolvimento Humano Municipal 2010 e consumo per capita de água para o estado da Bahia e suas mesorregiões.

\begin{tabular}{|c|c|c|c|c|}
\hline & Quantidade de & municípios & r de & Classificação da \\
\hline & Existentes & Analisados & Pearson & correlação \\
\hline Extremo oeste baiano & 24 & 21 & $+0,263$ & Fraca positiva \\
\hline Vale São-Francisco da Bahia & 27 & 17 & $+0,157$ & Fraca positiva \\
\hline Centro Norte da Bahia & 80 & 79 & $+0,326$ & Fraca positiva \\
\hline Nordeste Baiano & 60 & 60 & $+0,305$ & Fraca positiva \\
\hline Metropolitana de Salvador & 38 & 38 & $+0,592$ & Moderada positiva \\
\hline Centro Sul Baiano & 118 & 111 & $+0,107$ & Fraca positiva \\
\hline Sul baiano & 70 & 67 & $+0,499$ & Fraca positiva \\
\hline Estado da Bahia & 417 & 393 & $+0,391$ & Fraca positiva \\
\hline
\end{tabular}

Fonte: elaboração própria.

De acordo com os resultados apresentados na Tabela 2, observa-se que tanto no panorama geral para todo o estado da Bahia quanto para as análises realizadas para cada mesorregião do estado, obtiveramse valores do coeficientes de Pearson que classificam as correlações como positivas, ou seja: quanto mais elevado o IDHM, maior o consumo per capita de água. Porém, essa influência positiva entre os parâmetros foi classificada como fraca, com exceção da mesorregião metropolitana de Salvador, na qual a tendência positiva de linearidade entre os parâmetros apresentou-se como moderada, de acordo com a categorização de Santos (2007) constante na Tabela 1.
Correlação positiva entre IDHM e consumo per capita de água também foi observada em municípios dos estados de Minas Gerais (FERNANDES NETO et al., 2005) e de Goiás (AQUINO et al., 2017), sugerindo que quanto maior a qualidade de vida da população, maior é seu consumo médio diário de água.

Para uma visão mais ampla acerca dos valores de IDHM e consumo de água de cada município analisado, apresenta-se na Figura 2 a dispersão dos pares de valores dessas variáveis, categorizados para cada mesorregião do estado da Bahia.

Figura 2. Dispersão dos dados de índice de desenvolvimento humano (IDHM) e de consumo per capita de água em 2010 dos municípios estudados, por mesorregião.

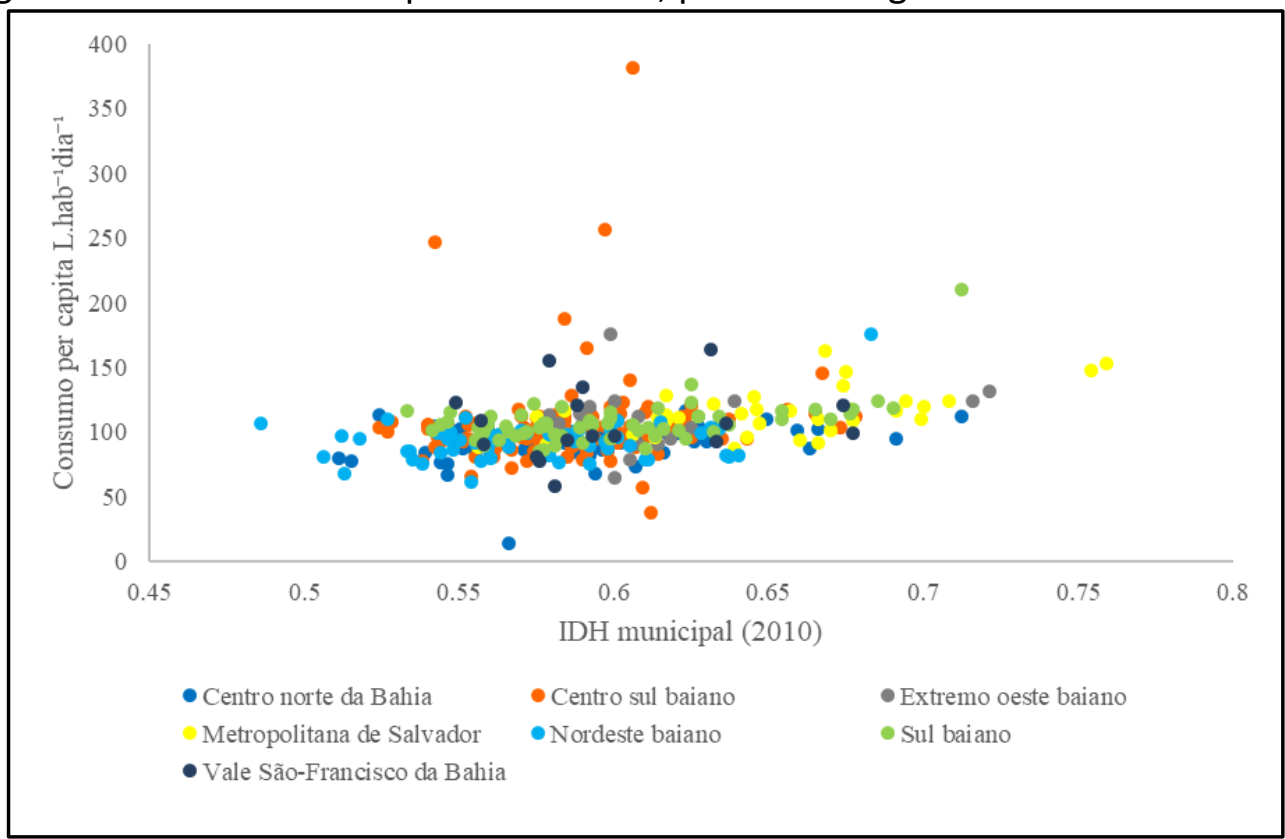

Fonte: Atlas Brasil (2020); SNIS (2020b). 
Pode-se observar na Figura 2 que não há nítido ordenamento quanto ao comportamento de consumo de água em função do desenvolvimento humano, corroborando os valores positivos e, em sua maioria, de fraca correlação dos coeficientes de Pearson já apresentados na Tabela 2. Observa-se ainda que os municípios que apresentaram valores mais elevados de consumo diário médio per capita de água são pertencentes à mesorregião Centro sul baiano, sem apresentar, entretanto, os valores mais elevados de IDHM dos dados obtidos.

\subsection{Influência do PIB per capita no consumo per capita de água}

Especificamente na correlação entre os dados anuais do produto interno bruto per capita e do consumo per capita de água de cada município entre os anos de 2002 e 2016, 23 dos 417 municípios do estado apresentaram menos que 5 pares de valores PIB versus consumo de água e, por esse motivo, não tiveram sua correlação linear de Pearson realizada.

Desta forma, foram feitas nesta etapa 394 análises de correlação, uma para cada município com histórico suficiente de dados. Conforme já explanado na metodologia, a análise dessas correlações foi categorizada por mesorregião, visto que a discussão individual de 394 valores do coeficiente de Pearson é impraticável.

Dos 23 municípios que não tiveram dados suficientes para análise da correlação linear existente entre PIB e consumo de água, 12 são pertencentes à mesorregião Centro Sul Baiano: Abaíra, Belo Campo, Boquira, Dom Basílio, Igaporã, Matina, Novo Horizonte, Piatã, Riacho de Santana, Ribeirão do Largo, Sebastião Laranjeiras e Urandi; 1 do Extremo oeste baiano: Cocos; 1 do Nordeste Baiano: Araças; 2 do Sul baiano: Barro Preto e Jussari e mais 7 do Vale São-Francisco da Bahia: Campo Alegre de Lourdes, Feira da Mata, Itaguaçu da Bahia, Pilão Arcado, Sento Sé, Serra do Ramalho e Sítio do Mato.

Além da quantidade de municípios analisados em cada mesorregião, na Tabela 3 se apresentam informações relativas aos coeficientes de correlação linear de Pearson ( $r)$, como seus valores mínimo ( $r$ mín), médio ( $r$ méd) e máximo ( $r$ máx), além da classificação de acordo com o critério de Santos (2007) para o valor médio obtido na mesorregião, visto que para cada município analisado foi obtido e classificado seu respectivo valor de $r$ de Pearson.

Tabela 3. Valores e classificação do coeficiente de Pearson ( $r$ ) entre o PIB per capita e o consumo per capita de água para o estado da Bahia e suas mesorregiões entre os anos de 2002 e 2016.

\begin{tabular}{|c|c|c|c|c|c|c|}
\hline & \multicolumn{2}{|c|}{ Quantidade de municípios } & \multirow{2}{*}{$\begin{array}{c}r \\
\text { mín }\end{array}$} & \multirow{2}{*}{$\begin{array}{c}r \\
\text { méd }\end{array}$} & \multirow{2}{*}{$\begin{array}{c}r \\
\text { máx }\end{array}$} & \multirow{2}{*}{$\begin{array}{l}\text { Classificação da } \\
\text { correlação }\end{array}$} \\
\hline & Existentes & Analisados & & & & \\
\hline Extremo oeste baiano & 24 & 23 & $-0,919$ & $-0,011$ & $+0,916$ & Ínfima negativa \\
\hline Vale São-Francisco da Bahia & 27 & 20 & $-0,928$ & $+0,012$ & $+0,944$ & Ínfima positiva \\
\hline Centro Norte da Bahia & 80 & 80 & $-0,965$ & $-0,513$ & $+0,606$ & $\begin{array}{l}\text { Moderada } \\
\text { negativa }\end{array}$ \\
\hline Nordeste Baiano & 60 & 59 & $-0,922$ & $-0,405$ & $+0,869$ & Fraca negativa \\
\hline Metropolitana de Salvador & 38 & 38 & $-0,951$ & $-0,342$ & $+0,527$ & Fraca negativa \\
\hline Centro Sul Baiano & 118 & 106 & $-0,980$ & $-0,419$ & $+0,943$ & Fraca negativa \\
\hline Sul baiano & 70 & 68 & $-0,948$ & $-0,259$ & $+0,737$ & Fraca negativa \\
\hline Estado da Bahia & 417 & 394 & $-0,980$ & $-0,355$ & $+0,944$ & Fraca negativa \\
\hline
\end{tabular}

Fonte: elaboração própria.

Para os municípios de todo o estado da Bahia, verifica-se uma amplitude muito grande com alusão aos valores do coeficiente de correlação de Pearson entre PIB per capita e consumo per capita de água. O mínimo valor observado foi igual a -0,980, 
pertencente ao município de Ibicuí, mesorregião Centro Sul Baiano, enquanto o maior coeficiente de Pearson, +0,944, encontra-se na Mesorregião do Vale do SãoFrancisco da Bahia, no município de Barra.

Quanto se procede à análise pela média dos valores do coeficiente de Pearson para cada mesorregião, observa-se, ainda na Tabela 3, que apenas no Vale São-Francisco da Bahia obteve-se valor médio categorizado como positivo, estando todas as outras mesorregiões classificadas como ínfima negativa ou fraca negativa. Para o panorama geral do estado, a categorização também se enquadrou como fraca negativa.

Desta forma, resguardada a variação dos valores do coeficiente de correlação de Pearson, em média, existe uma relação linear negativa entre PIB e consumo de água, para o intervalo de tempo compreendido entre 2002 e 2016. Ou seja: quanto maior o PIB per capita municipal, menor é consumo de água por habitante do respectivo município.

Essa relação negativa também foi observada por Fernández-Durán e Lloret (2016) em 131 munícipios da região central oeste do México, nos quais os autores concluíram que um incremento de $10 \%$ no PIB per capita implicaria numa redução de $6,48 \%$ do consumo per capita de água.

Numa visão global do estado da Bahia, dos 394 municípios os quais tiveram suas influências do PIB no consumo de água quantificadas pelo coeficiente de Pearson, $34,8 \%$ tiveram essa influência classificada como moderada negativa, conforme se apresenta na Figura 3.

Figura 3. Distribuição entre as classificações do coeficiente de Pearson entre PIB e consumo per capita de água dos municípios estudados.

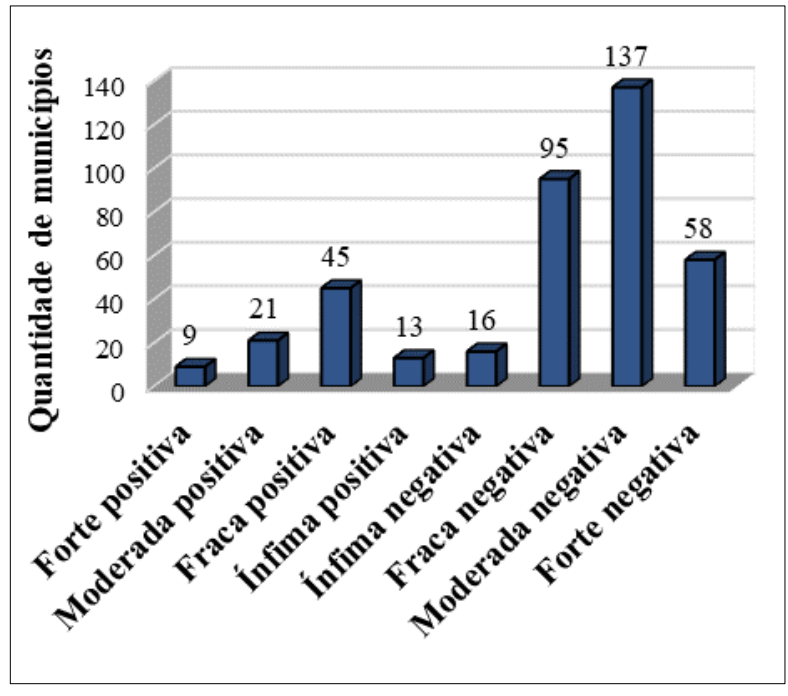

Fonte: elaboração própria.

Ademais, apenas 88 desses municípios apresentaram coeficientes cujos valores categorizaram-se como positivos, evidenciando que, no período de 2002 a 2016, em apenas 22,3\% dos municípios do estado a influência do PIB foi diretamente proporcional ao consumo per capita de água.

Situação similar à relação positiva e direta observada em apenas 88 dos 394 municípios baianos analisados pela presente pesquisa foi obtida por modelos matemáticos lineares e logarítmicos para dados de renda e de consumo de água do município de Belo Horizonte, capital do estado de Minas Gerais (DIAS et al., 2005). Semelhantemente, Silva, Silva e Chichorro (2008) ao estudarem possíveis influências de variáveis socioeconômicas no consumo per capita de água em Cuiabá, Mato Grosso, concluíram que quanto maior o rendimento nominal mediano mensal dos responsáveis por 
domicílio, maior o respectivo consumo de água.

Todavia, não obstante aos valores mesorregionais médios já discutidos, deve-se ressaltar a grande variabilidade do comportamento do consumo de água em função do PIB per capita, para os anos entre 2002 e 2016 nos 394 municípios analisados, segundo se apresenta espacialmente na Figura 4.

Figura 4. Valores do coeficiente de correlação de Pearson entre produto interno bruto e consumo per capita de água entre 2002 e 2016 para os municípios do estado da Bahia.

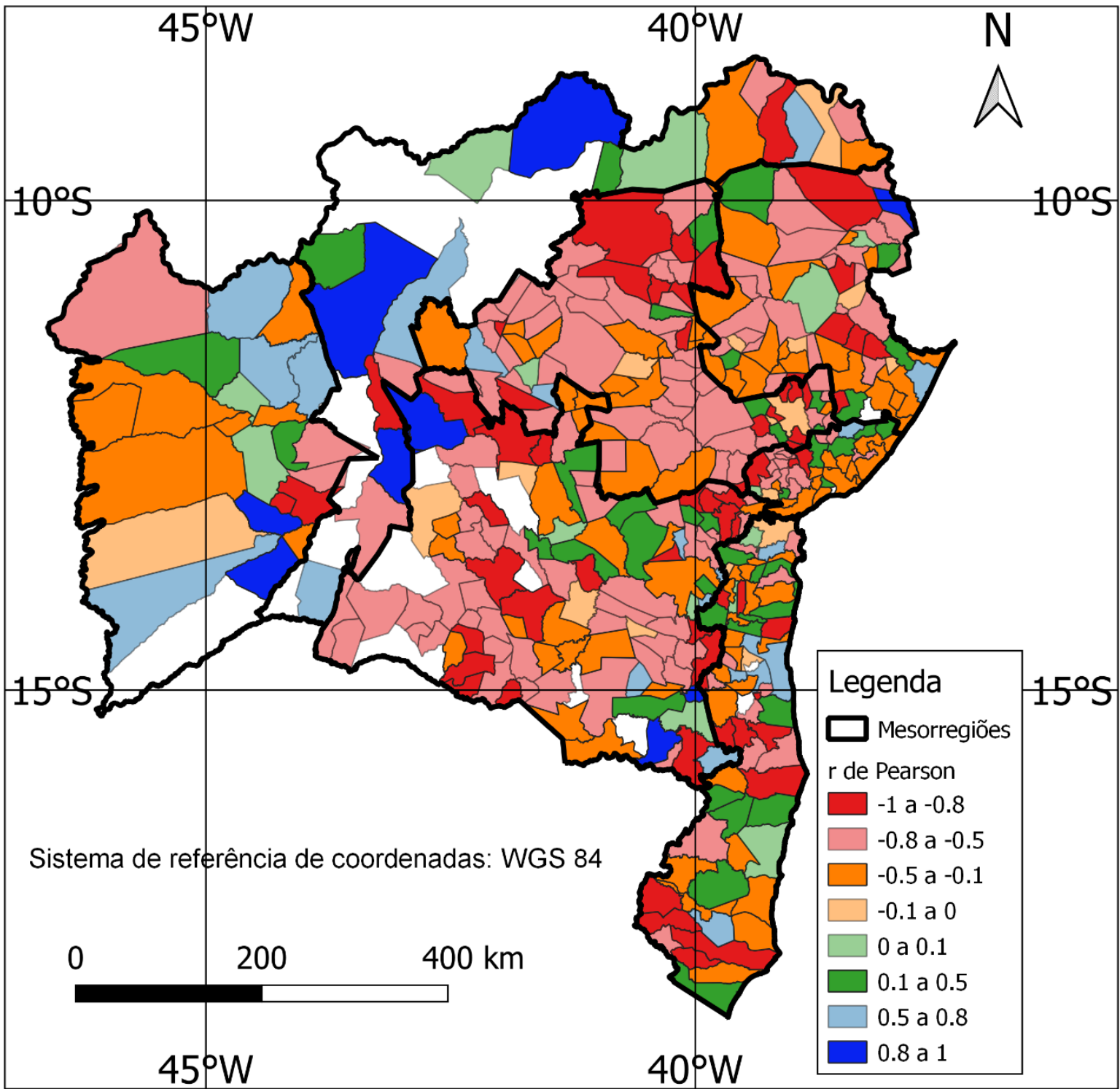

Polígonos em branco representam ausência de dados.

Fonte: elaboração própria, com base cartográfica extraída de IGEO (2020).

Conforme se pode depreender da análise da Figura 4, há predominância dos valores do coeficiente de Pearson compreendidos entre 0,8 e - 0,5, visto que 137 dos 494 municípios analisados enquadraram-se nessa faixa. Complementarmente, a segunda classe de valores mais frequente é a correspondente a fraca negativa, pela classificação de Santos (2007), compreendida entre $-0,5$ e $-0,1$. Por outro lado, a ocorrência de municípios cujos valores de $r$ os classificaram como correlação forte positiva, ou seja: $0,8 \leq r<1$ é muito pequena, o que reforça a tendência já discutida de maioria de valores negativos quanto à influência do PIB per capita no consumo de água, para o período de 2002 a 2016.

\section{CONCLUSÃO}


No período analisado, os municípios baianos apresentaram, de forma geral, correlação linear direta entre índice de desenvolvimento humano e consumo de água, sugerindo que municípios com valores mais elevados de IDHM apresentaram maior consumo de água no ano de 2010. Por outro lado, mesmo com grande variação de valores, observou-se que em média e no período de 2002 a 2016, os municípios analisados apresentaram menores consumos per capita de água, conforme maiores valores do seu PIB per capita.

A aplicação da correlação linear de Pearson para mensuração do grau e do sentido da relação existente entre o consumo per capita de água e os indicadores socioeconômicos municipais produto interno bruto e índice de desenvolvimento humano para os municípios do estado da Bahia no período considerado mostrou-se de grande utilidade, visto que os resultados advindos da interpretação dos seus coeficientes são de fácil entendimento e foram gerados por intermédio de uma ferramenta metodológica com simplicidade operacional. Essa ferramenta pode ser útil às companhias responsáveis pela operação dos serviços de abastecimento de água, bem como a outras instituições que realizem estudos de planejamento de ações correlatas ao saneamento básico.

\section{AGRADECIMENTOS}

Os autores agradecem ao campus Eunápolis e à Pró-Reitora de Pesquisa, PósGraduação e Inovação (PRPGI) do Instituto Federal de Educação, Ciência e Tecnologia da Bahia (IFBA), bem como à Fundação de Amparo à Pesquisa da Bahia (FAPESB) pela infraestrutura e pela concessão da bolsa de pesquisa que proporcionaram 0 desenvolvimento do presente estudo.

\section{REFERÊNCIAS}

ADAMS, A. A.; PABLOS, N. P. Factores que afectan la demanda de agua para uso doméstico en México. Región y sociedad, v. 22, n. 49, p. 3-16, 2010. Disponível em: http://www.scielo.org.mx/pdf/regsoc/v22n4 9/v22n49a1.pdf. Acesso em: 27 mai. 2020. ANA - AGÊNCIA NACIONAL DE ÁGUAS. Abastecimento Urbano de Água Bahia: resultados por município. Disponível em: http://atlas.ana.gov.br/Atlas/forms/analise/ Geral.aspx?est=13. Acesso em: 07 maio 2020.

AQUINO, D. S.; DIAS, L. K. A.; SOUZA, I. D.; CUNHA, A. P. A.; CORDEIRO, L. L. Influência de variáveis socioeconômicas municipais no consumo per capita de água. Revista AIDIS de Ingeniería y Ciencias Ambientales, v. 10, n. 1, p. 104-112, 2017. Disponível em: http://www.revistas.unam.mx/index.php/aid is/article/view/55224/52215. Acesso em: 25 maio 2020.

ATLAS BRASIL. Atlas do Desenvolvimento Humano no Brasil. Disponível em: http://www.atlasbrasil.org.br/2013/pt/o atla s/idhm/. Acesso em: 07 abr. 2020.

BONAMENTE, M. Statistics and Analysis of Scientic Data. 2. ed., New York: Springer, 2017. https://doi.org/10.1007/978-1-4939$\underline{6572-4}$

BRASIL. Presidencia da República. Constituição da República Federativa do Brasil de 1988. Brasília, DF: Presidência da República, 1988.

BRASIL. Lei no 11.445 , de 5 de janeiro de 2007. Estabelece diretrizes nacionais para o saneamento básico; altera as Leis nos 6.766, de 19 de dezembro de 1979, 8.036, de 11 de maio de 1990, 8.666, de 21 de junho de 1993, 8.987, de 13 de fevereiro de 1995; revoga a Lei no 6.528 , de 11 de maio de 1978; e dá outras providências. Diário Oficial da União. Brasília, 2007.

BRASIL. Ministério da Saúde. Portaria de Consolidação no 5, de 28 de setembro de 2017. Consolidação das normas sobre as ações e os serviços de saúde do Sistema Único de Saúde. Diário Oficial da União. Brasília, 2017. 
COSTA, C. L.; LIMA, R. F.; PAIXÃO, G. C.; PANTOJA, L. D. M. Avaliação da qualidade das águas subterrâneas em poços do estado do Ceará, Brasil. Semina: Ciências Biológicas e da Saúde, Londrina, v. 33, n. 2, p. 171-180, 2012. Disponível

em:

http://dx.doi.org/10.5433/1679-

0367.2012v33n2p171. Acesso em: 18 maio $2020 . \quad$ https://doi.org/10.5433/16790367.2012v33n2p171

DIAS, D. M.; MARTINEZ, C. B.; LIBÂNIO, M. Avaliação do impacto da variação da renda no consumo domiciliar de água. Engenharia Sanitária e Ambiental, v. 15, n. 2, p. 155-166, $2005 . \quad$ Disponível em: https://doi.org/10.1590/S1413-

41522010000200008. Acesso em: 27 maio $2020 . \quad$ https://doi.org/10.1590/S141341522010000200008

FERNÁNDEZ-DURAN, J. J.; LLORET, A. Consumo de agua y producto interno bruto en la cuenca Lerma-Chapala. Tecnología y Ciencias del Agua, v. 7, n. 4, p. 129-138, 2016. Disponível em: http://www.scielo.org.mx/pdf/tca/v7n4/200 7-2422-tca-7-04-00129.pdf. Acesso em: 19 mai. 2020.

FERNANDES NETO, M. L.; NAGHETTINI, M.; VON SPERLING, M.; LIBÂNIO, M. Assessing the relevance of intervening parameters on the per capita water consumption rates in Brazilian urban communities. Water Supply, v. 5, n. 1, p. 9-15, 2005. Disponível em: https://doi.org/10.2166/ws.2005.0002.

Acesso em: 27 mai. 2020. https://doi.org/10.2166/ws.2005.0002

FIGUEIREDO FILHO, D. B.; DA SILVA JUNIOR, J. A. Desvendando os Mistérios do Coeficiente de Correlação de Pearson ( $r$ ). Revista Política Hoje, v. 18, n. 1, p. 115-146, 2009. Disponível em:

https://periodicos.ufpe.br/revistas/politicaho je/article/viewFile/3852/3156. Acesso em: 15 maio 2020.
FUNASA.- FUNDAÇÃO NACIONAL DE SAÚDE. Manual de Saneamento. 5. ed. Brasília: Funasa, 2019.

GUERRERO, H. Excel Data Analysis: Modeling and Simulation. Cham, Switzerland: Springer, 2019. https://doi.org/10.1007/978-3-030$\underline{01279-3}$

HELLER, L. Abastecimento de água, sociedade e ambiente. In: HELLER, L.; DE PÁDUA, V. L. Abastecimento de água para consumo humano. 2. ed. Belo Horizonte: Ed. UFMG, 2010. v.1

IBGE - INSTITUTO BRASILEIRO DE GEOGRAFIA E eSTATÍSTICA. Panorama Bahia. Disponível em:

https://cidades.ibge.gov.br/brasil/ba/panora ma. Acesso em: 25 maio 2020a.

IBGE. INSTITUTO BRASILEIRO DE GEOGRAFIA E ESTATÍSTICA. Produto Interno Bruto - PIB. Disponível em: https://www.ibge.gov.br/explica/pib.php.

Acesso em: 18 mai. $2020 b$.

IGEO. INSTITUTO DE GEOCIÊNCIAS DA UFBA. Shapes Estado. Disponível em: http://www.twiki.ufba.br/twiki/bin/view/IGe o/ShapesEstado. Acesso em: 06 maio 2020.

LIBÂNIO, P. A. C.; CHERNICHARO, C. A. L.; NASCIMENTO, N. O. A dimensão da qualidade da água: avaliação da relação entre indicadores sociais, de disponibilidade hídrica, de saneamento e de saúde pública. Engenharia Sanitária e Ambiental, v. 10, n. 3, p. 219-288, 2005. Disponível em: http://dx.doi.org/10.1590/S1413-

41522005000300006. Acesso em: 25 maio $2020 . \quad$ https://doi.org/10.1590/S141341522005000300006

PNUD. PROGRAMA DAS NAÇÕES UNIDAS PARA O DESENVOLVIMENTO. Índice de Desenvolvimento humano municipal IDHM. Disponível em: https://www.br.undp.org/content/brazil/pt/ 
home/idh0/conceitos/o-que-e-o-idhm.html.

Acesso em: 25 abr. 2020.

ROCHA, H. M.; CABRAL, J. B. P.; BATISTA, D. F.; OLIVEIRA, L. G. Avaliação físico-química e microbiológica das águas da bacia hidrográfica do rio Paraíso Jataí-GO. Geosul, v. 34 , n. 72 , p. 51-74, 2019. Disponível em: https://doi.org/10.5007/1982-

5153.2019v34n72p51. Acesso em: 19 maio $2020 . \quad$ https://doi.org/10.5007/19825153.2019v34n72p51

SANTOS, C. Estatística descritiva:- manual de auto-aprendizagem. Lisboa: Edições Sílabo, 2007.

SEI. SUPERINTENDÊNCIA DE ESTUDOS ECONÔMICOS E SOCIAIS DA BAHIA. PIB municipal. Salvador, BA: SEI, [2017]. Disponível em: http://www.sei.ba.gov.br/index.php?option= com content\&amp;view=article\&amp;id=56 1\&amp;|temid=335. Acesso em: 07 abr. 2020.

SILVA, W. T. P.; SANTOS, A. A.; GOMES, L. A.; MUSIS, R. R. Quota per capita de água, fatores intervenientes e modelagem: estudo de caso para classes socioeconômicas de Cuiabá-MT. Sociedade \& Natureza, v. 20, n. 2, p. 219-230, 2008. Disponível em: https://doi.org/10.1590/S1982-

45132008000200013. Acesso em: 27 mai. $2020 . \quad$ https://doi.org/10.1590/S1982$\underline{45132008000200013}$

SILVA, W. T. P.; SILVA, L. M.; CHICHORRO, J. F. Gestão de recursos hídricos: perspectivas do consumo per capita de água em Cuiabá. Engenharia Sanitária e Ambiental, v. 13, n. 1, p. 8-14, 2008. Disponível em: https://doi.org/10.1590/S1413-

41522008000100002. Acesso em: 27 mai. $2020 . \quad$ https://doi.org/10.1590/S1413$\underline{41522008000100002}$

SOUZA, S. B. S.; FERREIRA N. C.; FORMIGA, K. T. M. Estatística espacial para avaliar a relação entre saneamento básico, IDH e remanescente de cobertura vegetal no estado de Goiás, Brasil. Revista Ambiente \& Água, v. 11, n. 3, p. 625-636, 2016. Disponível em: $\quad$ http://dx.doi.org/10.4136/ambiagua.1825. Acesso em: 21 maio 2020. https://doi.org/10.4136/ambi-agua.1825

SNIS, Sistema Nacional de Informações sobre Saneamento. Abastecimento de água - 2018. Disponível em: http://www.snis.gov.br/painel-informacoessaneamento-brasil/web/painelabastecimento-agua. Brasília: Ministério do Desenvolvimento Regional, 2018. Acesso em: 25 maio 2020a.

SNIS - SISTEMA NACIONAL DE INFORMAÇÕES SOBRE SANEAMENTO. Série histórica. Brasília: Ministério do Desenvolvimento Regional, 2018. Disponível em: http://app4.mdr.gov.br/serieHistorica/.

Acesso em: 07 abr. 2020b.

VON SPERLING, M. Introdução à qualidade das águas e ao tratamento de esgotos. 4. ed. Belo Horizonte: DESA/UFMG, 2014.

WASSERMAN, L. A. All of statistics: a concise course in statistical inference. New York: Springer, 2004. https://doi.org/10.1007/978$\underline{0-387-21736-9}$ 